\title{
ỨNG DỤNG PHƯƠNG PHÁP TƯ DUY THIẾT KẾ TRONG CÁC DƯ ÁN CỦA CDIO
}

\author{
Hồ Xuân Trường ${ }^{(*)}$ \\ (*) Thạc sĩ. Truờng Đại học Công nghệĐồng Nai. Email: hoxuantruong@dntu.edu.vn
}

DOI: $10.37550 /$ tdmu.CFR/2021.01.114

\section{Tóm tắt}

Đối với sinh viên theo học mô đun "Dụ án thiết kế và đổi mới" tại truờng Đại học Công nghệ Đồng Nai, có thể nhận thấy rằng bước khó nhất trong chuoong trình CDIO là buớc đầu tiên "hình thành ý tương". Phuơng pháp "Tu duy thiết kế" nhấn mạnh sụ hiểu biết sâu sắc về người tiêu dùng thông qua các cuộc khảo sát và quan sát chi tiết của người dùng, sau đó tiến hành phân tích dĩ liệu thu thập được. Phuong pháp tu duy thiết kế có thể giúp sinh viên trong bước đầu về "hình thành ý twởng” được hay không. Trong bài báo này sẽ mô tả về các thử nghiệm và sủ dụng phuơng pháp tu duy thiết kế trong viẹc hình thành ý tương của một dự án CDIO. Đồng thời nó cũng chỉ ra nhũng hạn chế và khó khăn ràng buộc của phương pháp này.

Tù̀ khóa: Tu duy thiết kế, đồng cảm, ý tuoơng, thực hành, sáng tạo

\section{1. Đặt vấn đề}

Kể từ khi phương pháp tiếp cận CDIO được áp dụng làm phương pháp giảng dạy, sinh viên thực hiện mô đun "Dự án Thiết kế và Đổi mới” ở năm thứ ba tại Trường Đại học Công nghệ Đồng Nai, đã có thể hình thành, thiết kế và thực hiện nhiều ý tưởng dự án, dựa trên bộ vi điều khiển.

Người ta đã quan sát thấy rằng bước khó khăn nhất trong quy trình $\mathrm{CDIO}$ là bước đầu tiên - "Conceive - hình thành ý tưởng". Trong số những vấn đề mà sinh viên gặp phải trong giai đoạn hình thành ý tưởng này là: 1 . Hạn chế về thời gian. 2 . Thiếu cách tiếp cận để hình thành và lựa chọn ý tưởng. 3. Thiếu kinh nghiệm thực tế để đưa ra đánh giá về tính hữu ích của một ý tưởng.

Phương pháp "Tư duy thiết kế" nhấn mạnh đến "sự hiểu biết sâu sắc về người tiêu dùng", thông qua các khảo sát và quan sát chi tiết người dùng và phân tích dữ liệu thu thập được. Phương pháp Tư duy thiết kế có thể sử dụng trong bước "hình thành ý tưởng” không?

Một nhóm sinh viên đa ngành đã trải qua "cuộc thử nghiệm" sử dụng phương pháp tư duy thiết kế để hiểu và nắm bắt được nhu cầu của người tiêu dùng, để tạo và lựa chọn ý tưởng của dự án. Khi sinh viên thấy được mức độ phù hợp với các dự án của họ, các dự án đó sẽ 
được tiếp tục như đồ án năm cuối trước khi ra trường. Bài báo này mô tả cách phương pháp "Tư duy thiết kế" được sử dụng để giúp sinh viên phát triển tốt sự hiểu biết về người dùng, hình thành được các ý tưởng dự án tốt. Nó cũng nêu bật một số hạn chế trong việc sử dụng phương pháp Tư duy Thiết kế trong các dự án CDIO.

\section{Mô đun "Dự án thiết kế và đổi mới” được dạy tại Trường Đại học Công nghệ Đồng Nai}

Mô đun "Dự án thiết kế và đổi mới" được giảng dạy cho sinh viên năm thứ ba trong ba ngành (Kỹ thuật Điện \& Điện tử, Kỹ thuật Máy tính và Kỹ thuật Điện tử \& Truyền thông) tại Đại học Công nghệ Đồng Nai.

Các sinh viên có 30 giờ trong 15 tuần để thực hiện một dự án CDIO, yêu cầu của dự án phải là một sản phẩm ứng dụng của vi điều khiển. Mỗi lớp khoảng 20 học sinh trước tiên sẽ được chia thành các đội gồm 4 hoặc 5 học sinh.

Học sinh sẽ xác định các lĩnh vực họ quan tâm, ví dụ: giúp đỡ người già hoặc người tàn tật hoặc ứng dụng năng lượng mặt trời. Sau đó, giảng viên sẽ giải thích tầm quan trọng của việc thực hiện một cuộc khảo sát để tìm ra những gì người dùng cần, thay vì chỉ đơn giản là giả định. Các sinh viên cũng sẽ được dạy một số kỹ thuật để thực hiện một cuộc khảo sát, ví dụ: phỏng vấn theo cặp (một người đặt câu hỏi, người kia ghi chép), câu hỏi nên kết thúc mở (không phải trả lời có/không)...

Hoạt động thực hiện khảo sát sẽ được giao cho học sinh. Nhóm dự án sẽ phải sắp xếp thời gian ngoài giờ học của chương trình học để thực hiện phỏng vấn một nhóm người dùng, nhằm mục tiêu tìm hiểu xem họ cần gì.

Các sinh viên cũng sẽ được yêu cầu nghiên cứu "thị trường sản phẩm" những sản phẩm hoặc dịch vụ nào đã có trên thị trường, để phục vụ nhu cầu được xác định trong cuộc khảo sát? Làm thế nào để "chiến lược đại dương xanh" được sử dụng để vượt qua các đối thủ cạnh tranh.

Với một số kiến thức về cung và cầu của thị trường, tiếp theo sinh viên sẽ suy nghĩ để đưa ra ý tưởng dự án khả thi trước khi trình bày với giảng viên và các bạn cùng lớp. Ý tưởng thường sẽ được trình bày bằng một "bản phác thảo ý tưởng" (Hình 1) để minh họa sản phẩm được tạo ra như thế nào. Các sinh viên sẽ trình bày về "quá trình sử dụng của người dùng" để cho thấy một người dùng thông thường sẽ sử dụng sản phẩm này như thế nào.

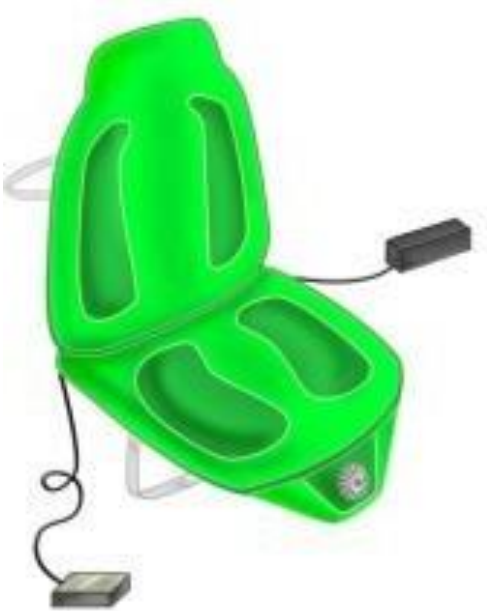

Hinh 1. Phác thảo ý tưởng chế tạo ghế rung

Sau giai đoạn "hình thành ý tưởng" này, sinh viên sẽ chuyển sang giai đoạn "thiết kế", trong giai đoạn này sinh viên kỹ thuật sẽ quay lại "lĩnh vực chuyên môn", vẽ sơ đồ khối và 
sơ đồ mạch cho phần cứng và thiết kế phần mềm của họ. Trong thời gian này, họ cũng sẽ được yêu cầu vạch ra "kế hoạch triển khai", để có thể tạo ra một nguyên mẫu đơn giản, hoạt động trong 5 hoặc 6 tuần tới, trong giai đoạn "thực hiện".

Trong khoảng thời gian giới hạn dành cho dự án, một lớp điển hình gồm 5 đội có thể tạo ra những thứ sau:

- “Cửa được bảo vệ bằng mật khẩu” - một mật khẩu chính xác phải được nhập qua bàn phím để cuộn dây điện từ (dùng khóa cửa) được tắt.

- "Đồng hồ báo thức" - khi chuông báo thức kêu, bốn đèn LED sẽ sáng theo một trình tự ngẫu nhiên và bạn bè buồn ngủ phải nhấn bốn nút theo cùng một trình tự để tắt chuông báo thức.

- "Đèn chỉ báo sức chứa của buồng vệ sinh" - đèn LED màu đỏ có nghĩa là buồng có người, đèn LED màu xanh lá cây có nghĩa là còn trống và tổng số buồng có sẵn được chỉ ra bên ngoài phòng vệ sinh để thuận tiện cho người sử dụng.

- "Gậy dành cho người mù" - một tiện ích cảnh báo/phát hiện chướng ngại vật dành cho người khiếm thị.

- "Ghế rung” - khi ngồi vào một thời gian, sẽ rung lắc để nhắc nhở người sử dụng không ngồi bàn quá lâu.

Khó khăn ở bước “C”" (Conceiving - hình thành ý tưởng) trong một dự án của CDIO:

Người ta đã quan sát thấy rằng bước "phức tạp" nhất trong quy trình CDIO là bước đầu tiên - "hình thành ý tưởng". Làm thế nào để các học sinh 18/19 tuổi, với kinh nghiệm sống còn hạn chế, đưa ra những ý tưởng khả thi về mặt kỹ thuật nhưng lại được người khác "muốn" hoặc “cần"? điều đó là không dễ để đưa ra đánh giá về tính hữu dụng của một ý tưởng. Trong số những vấn đề khác mà sinh viên phải đối mặt trong giai đoạn "hình thành ý tưởng" là: thời gian hạn chế và thiếu phương pháp tiếp cận có cấu trúc để hình thành và lựa chọn ý tưởng.

Hạn chế về thời gian là do chỉ có tổng cộng 30 giờ trong 15 tuần để làm việc cho dự án CDIO, với một phần lớn thời gian (hơn 20 giờ) cần thiết cho bước "I" (Implementation Thực hiện). Thực hiện liên quan đến chế tạo phần cứng, lập trình vi điều khiển, giao diện và xử lý sự cố. Còn lại rất ít thời gian cho giai đoạn "C" (Conceive) và "D” (Design)

Cũng không dễ để hướng dẫn một vài nhóm sinh viên trong lớp thông qua nghiên cứu người dùng, hình thành và lựa chọn ý tưởng chính bởi vì sinh viên có các lĩnh vực quan tâm rất khác nhau - một nhóm có thể quan tâm đến việc giúp đỡ người già trong khi nhóm khác có thể quan tâm giúp đỡ những người đi đường... Do đó, việc thực hiện khảo sát người dùng phần lớn thuộc về học sinh. Điều đó dẫn đến câu hỏi: Phương pháp Tư duy thiết kế có thể được sử dụng trong bước "hình thành ý tưởng" không?

\section{Phương pháp tư duy thiết kế}

\subsection{Khái quát về phưong pháp tư duy thiết kế}

“Tư duy thiết kế" thực sự là gì? Nếu bạn tra Google về thuật ngữ này thì rất có thể bạn sẽ thấy Stanford's D School hoặc Tim Brown... Tư duy thiết kế áp dụng một quy trình linh 
hoạt để giải quyết nhiều vấn đề khác nhau. Mặc dù không có định nghĩa duy nhất cho nó, nhưng những ưu điểm của nó sẽ được mô tả bên dưới:

_ “Tư duy thiết kế” có thể được mô tả như một cách thức sử dụng sự nhạy bén và sáng tạo của nhà thiết kế để đáp ứng nhu cầu của mọi người tiêu dùng với những gì khả thi về mặt công nghệ và chiến lược kinh doanh, nó có thể chuyển đổi thành giá trị khách hàng và cơ hội thị trường". Giám đốc điều hành Tim Brown, IDEO [2].

-Nó là một phương pháp luận để giải quyết các vấn đề hoặc vấn đề thực tế, sáng tạo. Đó là khả năng cần thiết để kết hợp sự đồng cảm, sáng tạo và hợp lý để đáp ứng nhu cầu của người dùng.

-Tư duy Thiết kế là một quá trình sáng tạo dựa trên việc "xây dựng" các ý tưởng. Không có đánh giá nào sớm về Tư duy thiết kế. Điều này khuyến khích tối đa đầu vào và tham gia vào các giai đoạn ý tưởng và nguyên mẫu. [3], [4], [5].

Tại Đại học Công nghệ Đồng Nai, một biểu đồ của phương pháp Tư duy thiết kế (Hình 2) có các bước chính sau: Đồng cảm, Ý tưởng \& Vật mẫu thử nghiệm. Nó nhấn mạnh đến "sự hiểu biết sâu sắc về người tiêu dùng", thông qua các cuộc khảo sát - quan sát chi tiết về người tiêu dùng và phân tích dữ liệu thu thập được.

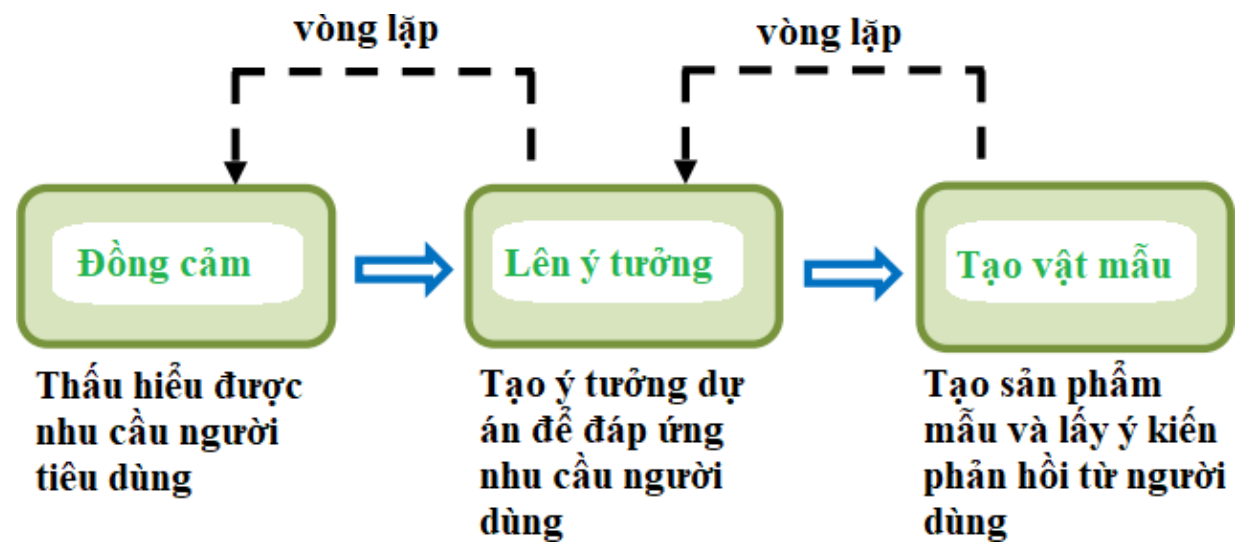

Hình 2. Các bước trong Tư duy thiết kế

Trong bước “thấu hiểu tâm lý người dùng”, nhóm các nhà thiết kế (hoặc kỹ sư) sẽ cố gắng tìm hiểu nhu cầu của người tiêu dùng về loại sản phẩm hoặc dịch vụ mà người dùng thực sự cần thiết. Điều này được thực hiện thông qua một số kỹ thuật như khảo sát và quan sát. Sau khi hiểu rõ các yêu cầu của người dùng, nhóm sẽ chuyển sang bước lên ý tưởng và đề xuất các giải pháp khả thi có thể giúp giải quyết vấn đề của người dùng. Bản phác thảo ý tưởng có thể được vẽ lên để nắm bắt các ý tưởng.

Thông thường, các giải pháp được đề xuất sẽ cho ra một Vật mẫu đầu tiên "mẫu chưa hoàn thiện" sau đó được trình bày cho người dùng để lấy ý kiến phản hồi. Các vật mẫu đầu tiên cho phép các ý tưởng không phù hợp bị loại bỏ sớm, khi đó chi phí đầu tư cho các thử nghiệm thất bại vẫn còn đang thấp.

Các mẫu đầu tiên không nhất thiết phải hoạt động ngay tại thời điểm này: Nó dùng cho việc giao tiếp trao đổi với khách hàng để lấy ý kiến về sản phẩm hoặc dịch vụ cuối cùng sẽ được sử dụng như thế nào là phù hợp nhất. Nó thậm chí có thể ở dạng video, tiểu phẩm, 
truyện tranh hoặc đơn giản là một bản phác thảo hay.

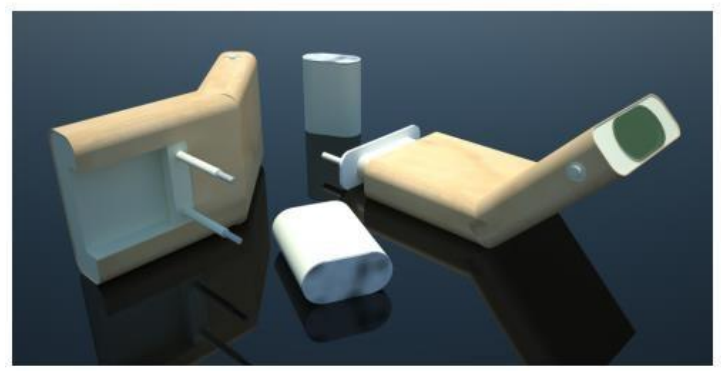

Hinh 3. Mẫu thư nghiệm bằng nhụa

Các phản hồi của người tiêu dùng sẽ được sử dụng để điều chỉnh lại các giải pháp được đề xuất. Sau đó, nhóm sẽ tiếp tục xây dựng một vật mẫu hữu dụng hơn trước khi đưa ra thị trường người tiêu dùng lần tiếp theo để thử sản phẩm hoặc dịch vụ.

Phương pháp tư duy thiết kế là một quá trình lặp đi lặp lại. Ví dụ: nếu trong quá trình "Ideation" nhóm phát hiện ra rằng họ không thực sự có đủ hiểu biết về các yêu cầu của người dùng để đề xuất một giải pháp tốt, họ có thể phải lặp lại các nghiên cứu về "tâm lý người dùng”. Như đã mô tả ở trên, phương pháp Tư duy Thiết kế được đơn giản hóa và đóng góp cho các bước C \& D trong một dự án CDIO (Hình 4).

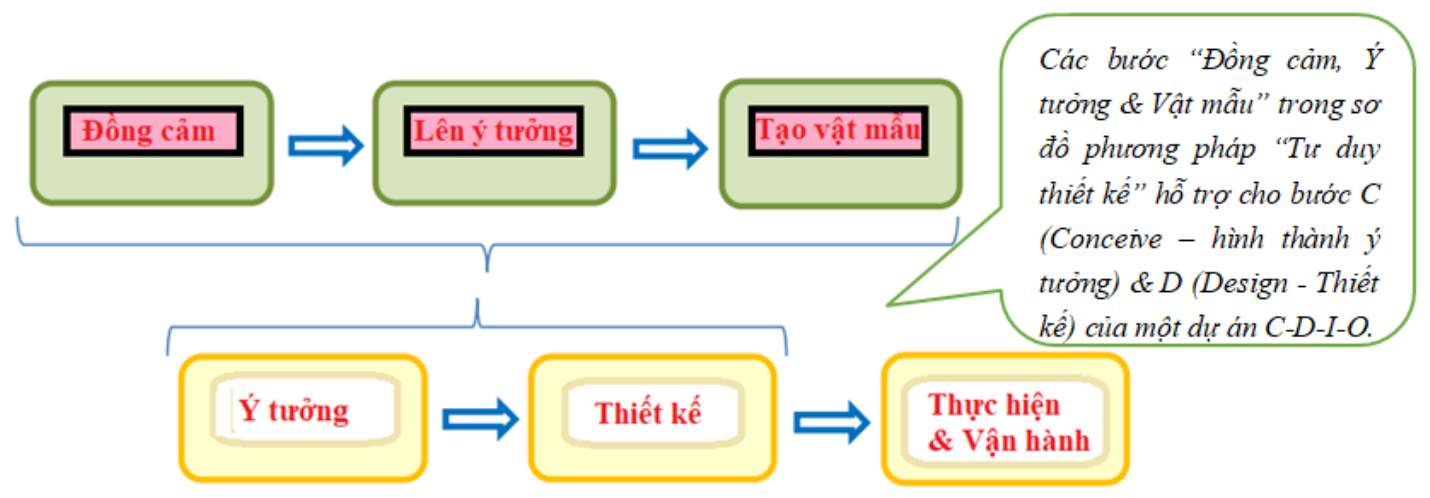

Hình 4. Tu duy thiết kế trong CDIO

\subsection{Sử dụng phuơng pháp "tư duy thiết kế" để giúp sinh viên hình thành được ý tương của một dụ án CDIO}

Vào tháng 8 năm 2016, 80 sinh viên (và một số giảng viên) đến từ ba ngành khác nhau (điện - điện tử; cơ khí và thiết kế) đã cùng nhau tham dự Hội thảo "Tư duy Thiết kế" kéo dài 4 ngày. Bốn mươi người lớn tuổi từ 50 tuổi trở lên cũng được mời làm đối tượng khảo sát nhu cầu. Chủ đề của hội thảo là "Ngôi nhà mơ ước". Mục đích của hội thảo này là để sinh viên từ các ngành khác nhau đến với nhau và sử dụng phương pháp "Tư duy thiết kế" để giải quyết các vấn đề thực tế. Có 80 sinh viên (và 40 người lớn tuổi) đã thành lập tổng cộng 20 nhóm dự án đa ngành, mỗi nhóm có 4 sinh viên (và 2 người lớn tuổi và một giảng viên làm hỗ trợ viên).

Trong bước “Thấu hiểu tâm lý người dùng” có các hoạt động sau được thực hiện: 
-Người dùng đã tạo được "bảng tâm trạng” (với hình ảnh và từ được cắt ra từ các tạp chí và dán chúng lên bìa cứng) để thể hiện ý tưởng của họ về ngôi nhà mơ ước.

-Các nhóm dự án đã đến nhà của những người lớn tuổi để quan sát môi trường sống của họ.

-Các nhóm dự án đã phỏng vấn những người dùng khác, ví dụ: người lớn tuổi tại những nơi mà người già thích đi dạo.

Sau tất cả các công việc "lên bảng tâm trạng, phỏng vấn và quan sát", các nhóm dự án bắt đầu đến bước tiếp theo "Lên ý tưởng". Họ phân tích dữ liệu thu thập được (quan sát, nhận xét, thông tin chi tiết,...) và suy nghĩ để đưa ra các ý tưởng dự án có thể giải quyết các vấn đề mà những người lớn tuổi nêu ra để tìm kiếm một "Ngôi nhà mơ ước". Các ý tưởng được ghi lại dưới dạng bản phác thảo ý tưởng, truyện tranh, video, vật mẫu bằng bìa cứng và được trình bày để được nhận xét, góp ý.

Sau 4 ngày thực hiện, sinh viên và giảng viên của 3 ngành tiếp tục họp 4 giờ mỗi tuần (trong hơn 2 tháng) để hoàn thiện các ý tưởng của dự án. Một điều nổi bật trong quá trình này là: Phương pháp luận của Tư duy Thiết kế không hướng đến sự kết luận nhanh chóng, tức là nó không cố gắng đưa ra ngay "giải pháp" một cách nhanh chóng. Nó khuyến khích bạn thỉnh thoảng nhìn lại một bước và tự hỏi "đó có phải là điều người dùng thực sự mong muốn không?"

Sau 2 tháng hoàn thiện ý tưởng, các sinh viên và giảng viên từ 2 ngành kỹ thuật đã tập hợp lại thành 6 nhóm dự án để làm việc trên các vật mẫu thực hiện dựa trên 6 ý tưởng được chọn trong hơn 2 tháng dưới đây:

-Tủ lạnh nằm ngang - cho phép người cao tuổi lấy đồ trong tủ lạnh mà không cần cúi người, vì cúi quá nhiều sẽ khiến người già gặp vấn đề về lưng.

-Một công cụ tìm kiếm đồ dùng - giúp người già (hay quên) xác định được vị trí đồ bị thất lạc trong nhà.

-Gương gia đình - cho phép các thành viên trong gia đình để lại tin nhắn video cho những người khác, trước khi rời khỏi nhà.

-Một khu vườn công nghệ - khai thác năng lượng mặt trời và cho phép tưới cây thường xuyên theo chương trình.

-Một "quái vật hút bụi" - cho phép người dùng chơi trò chơi quái vật hút bụi trong khi lau sàn. Điều này có thể sẽ cho phép người già chuyển giao công việc lau nhà hàng ngày cho các cháu của mình.

-Bộ "cốc gia đình cùng nhau" - thắp sáng nhiều đèn LED hơn khi nhiều thành viên trong gia đình dùng bữa cùng nhau. Điều này giúp nhắc nhở các thành viên trong gia đình tầm quan trọng của việc dùng bữa cùng nhau.

Vì các sinh viên kỹ thuật có thể thấy mức độ phù hợp của các ý tưởng dự án của mình, các dự án năm thứ hai này của sinh viên được thực hiện như là đề tài tốt nghiệp năm cuối của họ. 
Trở lại câu hỏi: "Tu duy Thiết kế đã được sủ dụng nhu thế nào để giúp sinh viên hình thành ý tương dư án tốt hơn? ” Bảng sau đây tóm tắt sự khác biệt đặc trưng giữa các dự án được hình thành "có" và "không" sử dụng phương pháp Tư duy thiết kế:

\begin{tabular}{|c|c|}
\hline $\begin{array}{l}\text { Các dự án được hình thành do sinh viên } \\
\text { thuộc "Dự án đa ngành - dùng phương pháp tư } \\
\text { duy thiết kế" }\end{array}$ & $\begin{array}{l}\text { Các dự án được hình thành do sinh viên thuộc } \\
\text { "Dự án thiết kế và đổi mới" }\end{array}$ \\
\hline $\begin{array}{l}\text { Bằng chứng cho thấy việc nghiên cứu và phân tích } \\
\text { dữ liệu thu thập về người dùng: } \\
\text { - Rất nhiều thời gian cho việc xác định các vấn đề. } \\
\text { ví dụ: "Làm cái gì". Không có sự vội vàng để đi } \\
\text { đến giải pháp ví dụ: "làm thế nào". } \\
\text { • Thông tin chi tiết về các dự án được đưa ra sau } \\
\text { rất nhiều lần cân nhắc và lặp lại, dựa trên nhu } \\
\text { cầu và mối quan tâm của người dùng. } \\
\text { • Tập trung vào nhu cầu và mối quan tâm của } \\
\text { người dùng. } \\
\text { - Giải pháp cho vấn đề đòi hỏi kiến thức từ các } \\
\text { ngành khác nhau. ví dụ kỹ sư cơ khí. }\end{array}$ & $\begin{array}{l}\text { Thường thiếu bằng chứng về nghiên cứu và phân } \\
\text { tích dữ liệu về người dùng trực tiếp. } \\
\text { - Sự tập trung của vấn đề xảy ra quá nhanh. } \\
\text { - Thông tin chi tiết về các dự án được đưa ra sau } \\
\text { một buổi làm việc tập trung cao độ. } \\
\text { - Thường tập trung vào dự án mà học sinh có thể } \\
\text { làm trong thời gian giới hạn, với kiến thức và kỹ } \\
\text { năng hạn chế của hộ về bộ điều khiển vi mô. } \\
\text { - Giải pháp cho vấn đề có thể được cung cấp bởi } \\
\text { một mình các sinh viên thuộc nhóm ngành kỹ } \\
\text { thuật điện \& điện tử. }\end{array}$ \\
\hline
\end{tabular}

Bảng 1. Sụ khác biệt đặc trung giữa các dụ án được hình thành "có” và "không” sủ dụng phuoong pháp Tu duy thiết kế

Bảng sau đây sẽ tóm tắt sự khác biệt giữa mô-đun "Dự án Thiết kế và Đổi mới” thông thường và quá trình thử nghiệm của "Dự án Đa ngành/Hội thảo Tư duy thiết kế":

\begin{tabular}{|c|c|c|}
\hline & $\begin{array}{l}\text { Dự án đa ngành/Hội thảo tư duy thiết } \\
\text { kế }\end{array}$ & Dự án Thiết kế và đổi mới \\
\hline $\begin{array}{l}\text { Thời gian cho toàn bộ } \\
\text { dự án }\end{array}$ & $\begin{array}{l}4 \text { ngày (chủ nhật) }+4 \text { giờ x } 15 \text { tuần học }+ \\
\text { thời gian thực hiện đề tài năm cuối }(6 \\
\text { tháng) }\end{array}$ & 30 giờ trong 15 tuần học \\
\hline $\begin{array}{l}\text { Thời gian dành cho } \\
\text { việc hình thành ý tưởng } \\
\text { dự án }\end{array}$ & 4 ngày (chủ nhật) + 4 giờ x 8 tuần học. & $\begin{array}{l}\text { Trong } 4 \text { tuần học, sinh viên được } \\
\text { hướng dẫn trong các bài học theo } \\
\text { lịch trình, các bài tập sẽ làm ngoài } \\
\text { giờ học }\end{array}$ \\
\hline "Chủ đề" cho dự án? & $\begin{array}{l}\text { Một chủ đề như "Ngôi nhà mơ ước" được } \\
\text { đưa ra. }\end{array}$ & $\begin{array}{l}\text { Sinh viên được phép tự do lựa chọn } \\
\text { các lĩnh vực mà họ quan tâm. }\end{array}$ \\
\hline $\begin{array}{l}\text { Hướng dẫn/trợ giúp } \\
\text { trong bước "hình thành } \\
\text { ý tưởng" }\end{array}$ & $\begin{array}{l}\text { Có sự sắp xếp công việc thực hiện thu } \\
\text { hút mục tiêu là người tiêu dùng. Mỗi đội } \\
\text { có một giảng viên làm người } \\
\text { điều hành để hướng dẫn. }\end{array}$ & $\begin{array}{l}\text { Chỉ đưa ra tóm tắt về việc sinh viên } \\
\text { phải lập kế hoạch cho cuộc phỏng } \\
\text { vấn/khảo sát của họ sẽ được thực } \\
\text { hiện như thế nào. }\end{array}$ \\
\hline $\begin{array}{l}\text { Bản chất của nhóm dự } \\
\text { án }\end{array}$ & $\begin{array}{l}\text { Mỗi nhóm dự án về bản chất là đa ngành, } \\
\text { bao gồm một vài kỹ sư điện } \\
\text { - điện tử, một vài kỹ sư cơ khí và một vài } \\
\text { sinh viên thiết kế. }\end{array}$ & $\begin{array}{l}\text { Mỗi nhóm dự án bao gồm } 4 \text { hoặc } 5 \\
\text { sinh viên điện - điện tử }\end{array}$ \\
\hline
\end{tabular}

Bảng 2. "Dụ án Đa ngành/Hội thảo Tu duy thiết kế" và "Dụ án Thiết kế và Đổi mới"

Qua bảng này ta thấy sinh viên thuộc "Dự án đa ngành/Tư duy thiết kế" đã dành nhiều thời gian hơn để hiểu về người tiêu dùng và nhu cầu của họ. 
Các công việc cũng được thực hiện để sinh viên thu hút người tiêu dùng và để làm cơ sở khả thi cho các bước sau, sinh viên được yêu cầu làm việc về một chủ đề duy nhất "Ngôi nhà mơ ước". Các giảng viên (người hướng dẫn) làm việc chặt chẽ và hướng dẫn sinh viên thông qua các cuộc phỏng vấn/khảo sát và phân tích dữ liệu tiếp theo.

Trên thực tế mỗi nhóm sinh viên với đa ngành nghề sẽ giúp họ làm được nhiều thứ mới và sáng tạo hơn, làm cho các vấn đề về kỹ thuật trở nên dễ dàng giải quyết hơn. Ví dụ: nếu một sinh viên điện - điện tử không thể tạo ra một cấu trúc hoàn chỉnh, người bạn trong nhóm của họ từ ngành khác sẽ có thể giúp đỡ hoàn thiện nó.

Theo kết quả của những khác biệt này (có các nghiên cứu về sự đồng cảm để hiểu người dùng + có sự hỗ trợ và hướng dẫn + dự án được thực hiện với một nhóm đa ngành), các ý tưởng được hình thành bằng phương pháp "Tư duy thiết kế" giải quyết tốt hơn nhu cầu của người tiêu dùng, như được nêu trong Bảng 1 .

\section{Một số hạn chế của việc áp dụng phương pháp tư duy thiết kế}

Như đã thấy rõ từ cuộc thảo luận ở trên, việc sử dụng “Tư duy thiết kế” trong việc hình thành các ý tưởng dự án tốt đi kèm với một số hạn chế như:

- Hạn chế về mặt thời gian, đôi khi phải thuyết phục sinh viên bỏ một vài ngày nghỉ để ở lại trường và thực hiện "nghiên cứu tâm lý của người tiêu dùng" cho các dự án của họ. Những sinh viên này cũng phải dành thêm vài giờ mỗi tuần cho dự án CDIO. Thông thường, thời gian bổ sung phải được phân bổ trong các giờ giảng dạy trên lớp để sinh viên thực hiện nghiên cứu về người dùng.

- Cần bố trí sao cho các sinh viên từ các ngành khác nhau làm việc cùng nhau. Họ phải có số giờ chung theo bảng thời gian cho dự án CDIO. Tương tự như vậy, các giảng viên phải được lập bảng thời gian. Phải có một địa điểm đủ rộng để chứa nhiều sinh viên như vậy.

- Cần tạo điều kiện nhiều hơn để các sinh viên thực hiện tham gia, phỏng vấn và quan sát người tiêu dùng. Công việc này chỉ có thể thực hiện được khi sinh viên làm việc trên cùng một dự án “chủ đề", ví dụ như dự án "Ngôi nhà mơ ước cho người lớn tuổi từ 50 trở lên”.

- Rất khó để có được cùng một nhóm người tiêu dùng (độ tuổi 50+) ở lại để thực hiện các khảo sát trong suốt dự án của sinh viên. Vì vậy, các mẫu thử được tạo chỉ có thể được đưa ra khảo sát cho những người dùng khác nhau để nhận xét và điều chinh.

Việc áp dụng thử nghiệm "Dự án đa ngành - Tư duy thiết kế", mặc dù đã thành công bước đầu, nhưng nó đòi hỏi cần rất nhiều nguồn lực về mặt thời gian, hậu cần và điều kiện thuận lợi. Phương pháp này sẽ khó để có thể cho phép một số lượng lớn sinh viên trải qua cùng một trải nghiệm. 


\section{Một số giải pháp khắc phục hạn chế khi áp dụng mô hình tư duy thiết kế tại Trường Đại học Công nghệ Đồng Nai}

Để cho phép nhiều sinh viên hơn được nhận thấy được lợi ích từ phương pháp "Tư duy thiết kế" trong các dự án của CDIO, Trường Đại học Công nghệ Đồng Nai đã bắt đầu phát triển "mô hình tư duy thiết kế" một cách toàn diện, xây dựng tập hợp các phương pháp như (Đồng cảm/Ý tưởng/Tạo mẫu thử) mà các giảng viên sẽ tập huấn cho sinh viên sử dụng trong các dự án $\mathrm{CDIO}$. Những công cụ này cho phép nhiều giảng viên nhanh chóng làm quen với phương pháp Tư duy thiết kế, và tạo điều kiện thuận lợi cho việc thực hiện dự án của sinh viên trong nhiều môi trường khác nhau. Một số giải pháp mà nhà trường đã đưa ra khắc phục các hạn chế khi áp dụng phương pháp này là:

- Để sinh viên có thể chủ động hơn về mặt thời gian để thực hiện mô hình tư duy thiết kế trong dự án của CDIO, nhà trường đã xây dựng hệ thống học trực tuyến Elearning giúp sinh viên có thể không cần dành nhiều thời gian trên lớp để học mà có thể xem lại buổi học vào một thời gian khác. Khi đó sinh viên có thể dành thời gian đó để thực hiện việc khảo sát "tâm lý của người tiêu dùng" tốt hơn.

- Sắp xếp thời khóa biểu của sinh viên và giảng viên phù hợp cho các khóa học có áp dụng phương pháp tư duy thiết kế.

- Xây dựng mô hình thư viện hiện đại cho phép một số lượng lớn sinh viên và giảng viên có môi trường thích hợp tham gia thực hiện các dự án CDIO tại đây.

- Xây dựng một khu "Sáng tạo và Khởi nghiệp" cho phép sinh viên có môi trường khảo sát nhu cầu người tiêu dùng thông qua việc trưng bày và bán các sản phẩm vật mẫu theo ý tưởng.

- Xây dựng phòng nghiên cứu cho sinh viên đa ngành, tại đây các nhóm sinh viên từ nhiều ngành khác nhau có thể làm việc cùng nhau để thực hiện các nghiên cứu theo mô đun dự án.

\section{Tài liệu tham khảo}

[1] TS. Hồ Tấn Nhựt, Đoàn Thị Minh Trinh (Biên dịch), "Cải cách và xây dựng chương trình đào tạo kỹ thuật theo phương pháp tiếp cận CDIO”, Nxb Đại học Quốc gia TP.HCM, 2009.

[2] TS. Hồ Tấn Nhựt , "The CDIO approach to engineering education: Introduction”, 2008.

[3] MELLES, Gavin, et al. "Problem finding through design thinking in education. In: Inquirybased learning for multidisciplinary programs: A conceptual and practical resource for educators", Emerald Group Publishing Limited, 2015.

[4] Crawley E, Malmqvist J, Ostlund S. and Brodeur D, "Rethinking Engineering Education: The CDIO Approach", Springer, 2007.

[5] DUNNE, David; MARTIN, Roger. and how it will change management education: An interview and discussion", Academy of Management Learning \& Education, 2006, pp 512-523. 
